\title{
Exploratory Behavior and Recognition Memory in Medial Septal Electrolytic, Neuro- and Immunotoxic Lesioned Rats
}

\author{
M. G. DASHNIANI ${ }^{1}$, M. A. BURJANADZE ${ }^{1}$, T. L. NANEISHVILI ${ }^{1,2}$, N. C. CHKHIKVISHVILI ${ }^{1}$, \\ G. V. BESELIA ${ }^{1}$, L. B. KRUASHVILI ${ }^{1,2}$, N. O. POCHKHIDZE ${ }^{1,3}$, M. R. CHIGHLADZE ${ }^{1,2}$ \\ ${ }^{1}$ I. Beritashvili Center of Experimental Biomedicine, Tbilisi, Georgia, ${ }^{2}$ St. Andrew the First-Called \\ Georgian University of Georgian Patriarchate, Tbilisi, Georgia, ${ }^{3}$ Ilia State University, Tbilisi, \\ Georgia
}

Received April 15, 2014

Accepted November 28, 2014

On-line March 24, 2015

\section{Summary}

In the present study, the effect of the medial septal (MS) lesions on exploratory activity in the open field and the spatial and object recognition memory has been investigated. This experiment compares three types of MS lesions: electrolytic lesions that destroy cells and fibers of passage, neurotoxic ibotenic acid lesions that spare fibers of passage but predominantly affect the septal noncholinergic neurons, and immunotoxin - 192 IgG-saporin infusions that only eliminate cholinergic neurons. The main results are: the MS electrolytic lesioned rats were impaired in habituating to the environment in the repeated spatial environment, but rats with immuno- or neurotoxic lesions of the MS did not differ from control ones; the MS electrolytic and ibotenic acid lesioned rats showed an increase in their exploratory activity to the objects and were impaired in habituating to the objects in the repeated spatial environment; rats with immunolesions of the MS did not differ from control rats; electrolytic lesions of the MS disrupt spatial recognition memory; rats with immuno- or neurotoxic lesions of the MS were normal in detecting spatial novelty; all of the MSlesioned and control rats clearly reacted to the object novelty by exploring the new object more than familiar ones. Results observed across lesion techniques indicate that: (i) the deficits after nonselective damage of MS are limited to a subset of cognitive processes dependent on the hippocampus, (ii) MS is substantial for spatial, but not for object recognition memory the object recognition memory can be supported outside the septohippocampal system; (iii) the selective loss of septohippocampal cholinergic or noncholinergic projections does not disrupt the function of the hippocampus to a sufficient extent to impair spatial recognition memory; (iv) there is dissociation between the two major components (cholinergic and noncholinergic) of the septohippocampal pathway in exploratory behavior assessed in the open field - the memory exhibited by decrements in exploration of repeated object presentations is affected by either electrolytic or ibotenic lesions, but not saporin.

\section{Key words}

Septohippocampal projections - Recognition memory • Exploratory behavior $\bullet$ Open field $\bullet$ Rat

\section{Corresponding author}

M. G. Dashniani, I. Beritashvili Center of Experimental Biomedicine, 14 Gotua Street, 0160, Tbilisi, Georgia. Fax: 995322373411. E-mail: m.dashniani@yahoo.com

\section{Introduction}

Several behavioral studies show that under conditions in which recognition memory has a spatial or temporal component, the hippocampus appears to be critical (Barbosa et al. 2012, Balderas et al. 2012, Barker and Warburton 2011). Evidence indicating that the medial septum (MS) is connected to the hippocampus (Mesulam et al. 1983) predicts that MS lesions should disrupt the recognition memory. MS lesions spare many of the efferent and afferent connections compromised by hippocampal formation damage or fimbria-fornix transection. Therefore, intact systems may be sufficient to support the hippocampal function in recognition memory. Septohippocampal projections are predominantly cholinergic and GABAergic (Rye et al. 1984), although 
there are some glutamatergic (Sotty et al. 2003) and neuropeptide projection neurons as well (Peterson and Shurlow 1992).

The data of different experimental approaches suggest that the septohippocampal cholinergic system is crucial for normal memory function (Chang and Gold 2003, Lecourtier et al. 2011). However, findings from experiments using the immunotoxin 192 IgG-saporin to produce selective lesions of cholinergic septal neurons frequently fail to cause any impairment in spatial learning in the water maze, spatial working memory in the radial arm maze, or contextual fear conditioning (Kirby and Rawlins 2003, Frick et al. 2004, Dashniani et al. 2009). These findings have raised the possibility that hippocampal ACh is not essential for all types of hippocampal-dependent memory.

Studies investigating the functional significance of noncholinergic MS neurons have been sparse. Ibotenic acid is a neuroexcitatory amino acid that functions as a NMDA and metabotropic glutamate receptor agonist. Ibotenic acid has been well known for its axonsparing excitotoxicity (Jarrard 1989) and its excitotoxic properties are confined to the somata of various neuron types (Choi et al. 1988). However, intraseptal administration of some excitotoxins preferentially damage noncholinergic basal forebrain neurons (Dunnett et al. 1991). Thus, according to immunohistochemical data, some authors describe that low doses of kainic or ibotenic acid predominantly destroy GABAergic neurons, while sparing most cholinergic cells (Cahill and Baxter 2001, Pang et al. 2001, Yoder and Pang 2005). In addition, it should be noted that despite the extensive damage to GABAergic septohippocampal neurons, spatial working and reference memory were unimpaired (Pang et al. 2001).

Although the importance of the septohippocampal projections in learning and memory is generally accepted (Myhrer 1989, Poucet 1989, Okada and Okaichi 2010), the degree to which damage to particular set of septohippocampal projections contributes to deficits within specific cognitive domains is less clear. This is due, in part, to the fact that different laboratories produce lesions in different ways, individual studies often lesion only one subset of septohippocampal projections rather than comparing lesions of different subsets. This makes it difficult to compare the degree of impairment produced by different lesions.

For this reason, in order to investigate the possible involvement the septohippocampal cholinergic and noncholinergic projections in spatial and object recognition memory, we have opted for a non-associative task where no explicit reward was present. This task consists of placing rat in an open field containing four objects and, after three sessions of habituation, examining their reactivity to object displacement (spatial novelty) and object substitution (object novelty). The purpose of this study was to investigate ability to acquire and use spatial or non-spatial information as well as to habituate exploratory activity over time in sham-operated, electrolytic, neuro- or immunotoxic MS lesioned rats.

\section{Methods}

\section{Subjects}

A total of 39 male Wistar rats, approximately 4 months of age and weighing 220-250 $\mathrm{g}$ at the start of experimentation served as subjects. Upon arrival, the rats were housed under controlled environment (temperature 20-22 ${ }^{\circ} \mathrm{C}$, humidity $-55-60 \%$ ). Each rat was then gently handled by the experimenter a few minutes per day for several days in experimental room to prevent neophobic reactions. The rats were housed in standard cages at a natural light/dark cycle and were tested during the light period. They had access to food and water ad libitum. The experiments have been conducted in accordance with the Guide for the Care and Use of Laboratory Subjects. Experimental protocol was approved by Animal Studies Committee of I. Beritashvili Center of Experimental Biomedicine.

\section{Surgery}

Rats were anaesthetized with i.p. injection of $4 \%$ chloral hydrate $(9 \mathrm{ml} / \mathrm{kg})$ and placed in a stereotaxic apparatus. For electrolytic lesions a stainless steel electrode $(0.15 \mathrm{~mm}$ in diameter $)$, insulated except at the tip, was inserted in the MS (AP - 0.7; ML - 0; DV - 6.5), according to Paxinos and Watson (1998) stereotaxic atlas; A $1.0 \mathrm{~mA}$ anodal current was passed through the electrode twice for $30 \mathrm{~s}$. Sham operations ( $\mathrm{n}=4$ rats) were performed by inserting the electrode at the same coordinates except that the depth was only $0.5 \mathrm{~mm}$ and electrolytic lesion was not produced. All injections of 192 IgG-saporin (1 mg/ml of phosphate-buffered saline, Advanced Targetting System, San Diego, USA) for immunolesion surgeries or mouse saporin (this product serves as a control for the immunotoxin) for control surgeries ( $\mathrm{n}=4$ rats) and ibotenic acid $(10 \mathrm{mg} / \mathrm{ml}$ of phosphate-buffered saline; SIGMA-ALDRICH) for 
neurotoxic lesion surgeries or phosphate-buffered saline for control surgeries ( $\mathrm{n}=4$ rats) were performed stereotaxically at two positions: $\mathrm{AP}-0.7 ; \mathrm{ML}-0 ; \mathrm{DV}-$ $7.8 \mathrm{~mm}(0.3 \mu \mathrm{l} ; 0.05 \mu \mathrm{l} / \mathrm{min})$ and $\mathrm{DV}-6.2 \mathrm{~mm}(0.2 \mu \mathrm{l}$; $0.05 \mu \mathrm{l} / \mathrm{min})$. The needle was left in place for an additional $9 \mathrm{~min}$ and $6 \mathrm{~min}$, respectively, after completion of the injection, to allow the toxin to diffuse from the injection site. All injections were made with a 1- $\mu 1$ Hamilton syringe with a microinjection pump (CMA 402 Syringe Pump, Sweden). The rats were allowed to recover from the surgery for two weeks before starting the behavioral experiments.

\section{Behavioral apparatus}

An open-field square arena $(65 \times 65 \times 75 \mathrm{~cm})$ enclosed by walls made from wood and illuminated by a $60 \mathrm{~W}$ light bulb mounted $1 \mathrm{~m}$ above the area was used for the behavioral test. The floor of the arena was divided into 16 equal squares by white lines. The walls inside the arena were surrounded with a white cloth to a height of $1.5 \mathrm{~m}$. The environment was uniform except for a one striped pattern poster $(30 \mathrm{~cm}$ wide and $60 \mathrm{~cm}$ high) attached to the white cloth (the white cloth prevent the rat from looking out into the room and thereby, to maximize attention to the object of stimuli). An overhead camera and a video recorder were used to monitor and record the animal's behavior for subsequent analysis. The objects to be distinguished were made of glass, plastic, or metal and existed in duplicate. The weight of the objects ensured that they could not be moved by the rats. As far as could be ascertained, the objects had no natural significance for the rats and they had never been associated with a reinforcer.

\section{Behavioral procedure}

Rats were individually given five 3-min sessions, each of which was separated by 24-h delay, during which subjects were returned to their home cage. In all sessions animals were gently placed in the center of the open field. During Session 1 four different (by color, shape and size) objects (A, B, C, D) were simultaneously present in the open field.

The rat was placed into the open field to familiarize it with the apparatus and to record the baseline level of locomotor activity and object exploration. All rats were given three habituation sessions. For Session 4, the spatial location of the object (B) was modified and in Session 5, one of the familiar nondisplaced object (C) was substituted with a new one (object E) at the same location. The time spent exploring the novel and familiar or displaced and nondisplaced objects was recorded in Sessions 4 and 5 for $3 \mathrm{~min}$.

In order to exclude the odor traces, after each session, each object was substituted with a new one of same color, shape and size. After each test, the open-field was cleaned with a solution of $20 \%$ ethanol and then dried with a cloth.

\section{Behavioral measures}

Locomotor activity was assessed by counting the number of grid crossed by each animal while moving in the open field. The decrease in the number of crossings between Session 1 and Session 3 was taken to be a measure of habituation to the environment. The habituation score (difference score) was defined by subtracting the number of crossings in Session 3 from the number of crossings during Session 1. The amount of time spent by each animal for the object exploration was recorded. Exploration was considered to be directing the nose at a distance $<2 \mathrm{~cm}$ to the object and/or touching it. The habituation score (difference score) was defined by subtracting the time spent for exploring four objects in Session 3 from the time spent for the same objects during Session 1 (Bolivar and Flaherty 2003, Lee et al. 2005). The larger the habituation score data, the greater the habituation. The rats' responses to the spatial change (in Session 4) and object novelty (in Session 5) were evaluated as discrimination indexes (DIs) that takes into account individual differences in the total amount of exploration (Dix and Aggleton 1999, Ennaceur and Delacour 1988). The following equation was used for displacement discrimination index, $\mathrm{DI}_{\mathrm{D}}: \mathrm{DI}_{\mathrm{D}}=\mathrm{t}_{\mathrm{D}} /\left(\mathrm{tN}_{\mathrm{D}}+\right.$ $t_{D}$ ), where $t_{D}=$ exploration time of the displaced objects and $\mathrm{tN}_{\mathrm{D}}=$ mean exploration time of the non-displaced objects. The object novelty discrimination index, $\mathrm{DI}_{\mathrm{N}}$ was calculated as: $\mathrm{DI}_{\mathrm{N}}=\mathrm{t}_{\mathrm{N}} /\left(\mathrm{t}_{\mathrm{F}}+\mathrm{t}_{\mathrm{N}}\right)$, where $\mathrm{t}_{\mathrm{N}}=$ exploration time of the novel object and $t_{F}=$ mean exploration time of the familiar objects (Niewiadomska et al. 2006).

Number of grid crossings and the amount of time spent by each animal for the object exploration were calculated manually by replaying the videotapes and inspecting the rat's behavior. Counts were performed by a person who was blind to the treatments and did not participate to animal testing.

\section{Histology}

At the end of the behavioral experiments MS lesioned and control rats were deeply anesthetized with 
pentobarbital and perfused through the ascending aorta with $300 \mathrm{ml}$ saline followed by $600 \mathrm{ml} \quad 4 \%$ paraformaldehyde in $0.1 \mathrm{M}$ phosphate buffer ( $\mathrm{pH}$ 7.4). The size and location of the electrolytic and ibotenic acid lesions were determined by microscopic examination of serial coronal sections $(25 \mu \mathrm{m})$ stained with cresyl violet. The immunotoxic lesions of MS were verified by observing decreased acetylcholinesterase (AChE) staining of the MS and hippocampal sections (hippocampal AChE is used as a quantitative measure of lesion extent). For this purpose brains (from four sham and four MS immunotoxic lesioned animals) were dissected out, postfixed at $4{ }^{\circ} \mathrm{C}$ for an additional $3-4 \mathrm{~h}$ in the same fixative, and stored frozen $\left(-70^{\circ} \mathrm{C}\right)$ until used. The brains were cut in coronal plan on a freezing microtome. The sections at several levels of the hippocampus and MS (20- $\mu \mathrm{m}$ thick) were placed in phosphate buffer for $30 \mathrm{~min}$ and taken on polylysinecoated slides. The immunostaining was run in a random sample from each group of animals. Fixed sections were stained with AChE (h-134, Rabbit polyclonal antibody) primary antibody and its detection system - ABC Staining System. Stained sections were analyzed with fluorescence optic microscope Leica MM AF. The cell counting was performed in the CA1 and CA3 areas. For this purpose the systematic random sampling was employed. The 2-dimensional counting grid $(250 \mu \mathrm{m} \mathrm{x}$ $250 \mu \mathrm{m})$ at the magnification $400 \mathrm{x}$ was used. Totally 6-10 sections of hippocampal and MS levels within experimental and control animals were selected. All reagents and buffers were received from Santa Cruz Biotechnology, Inc. (USA).
A

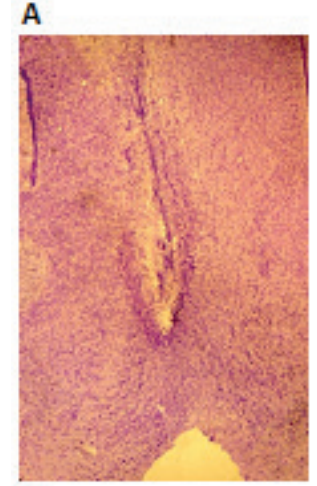

D
B

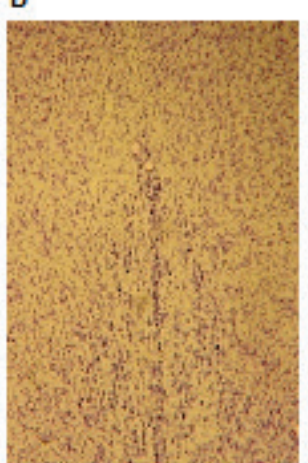

E

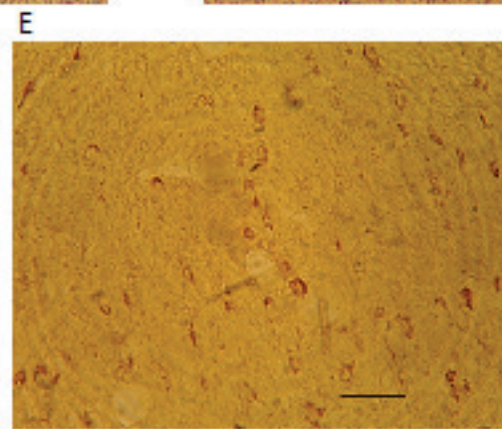

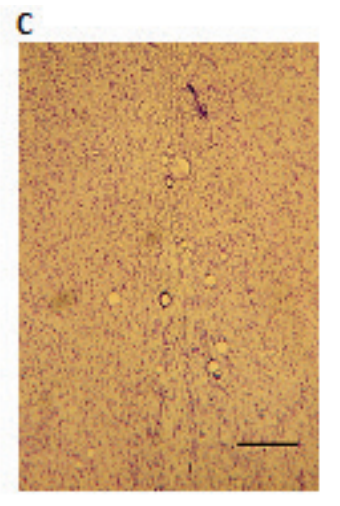

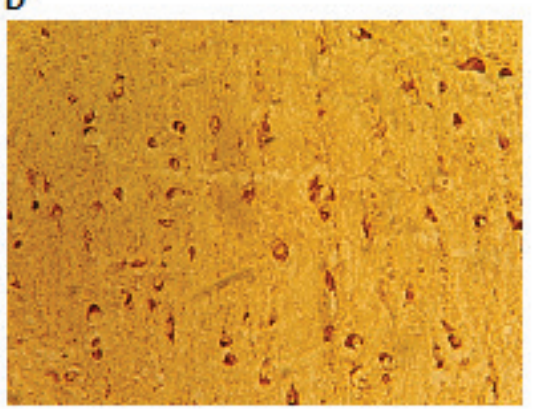

A
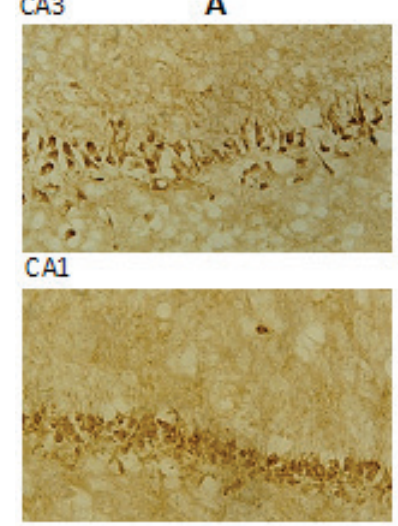

B
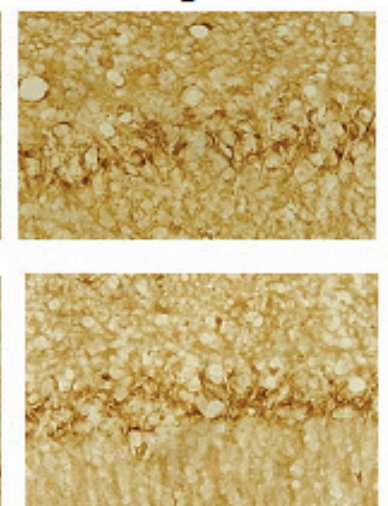

C
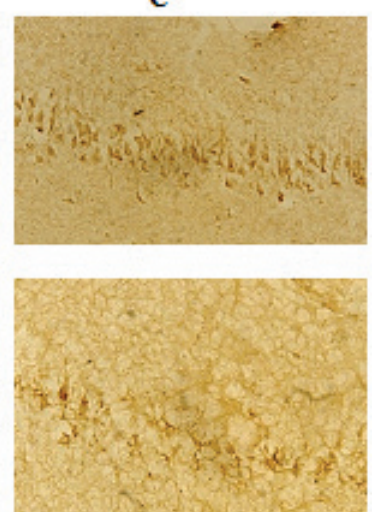

Fig. 1. A - the representative electrolytic lesion of the MS. B a sham lesion that left the medial septum intact, C - a representative ibotenic acid lesion that destroyed most of the MS neurons, D - AChE staining of the MS sections after injections of mouse saporin and $\mathbf{E}$ AChE staining of the MS sections after injections of $192 \mathrm{IgG}$-saporin into the MS. (Scale bar: $100 \mu \mathrm{m}$ for B and C; $50 \mu \mathrm{m}$ for $\mathrm{D}$ and $\mathrm{E}$ )

Fig. 2. $A C h E$ staining of the hippocampal (CA1, CA3) sections after injections of mouse saporin $(\mathbf{A})$ or 192 IgG-saporin (B) and ibotenic acid (C) into the MS. Scale bar, $50 \mu \mathrm{m}$ 
Table 1. Number of positive AChE cells in various hippocampal fields and in MS of rats (determined by immunostaining) and number of MS neurons determined by microscopic examination of serial coronal sections stained with cresyl violet.

\begin{tabular}{|c|c|c|c|c|}
\hline \multirow{3}{*}{ Group } & \multicolumn{3}{|c|}{ Number of positive $A C h E$ cells } & \multirow{3}{*}{$\begin{array}{c}\text { Number of medial septal } \\
\text { neurons (Nissl staining) }\end{array}$} \\
\hline & \multicolumn{2}{|c|}{ Hippocampal fields } & \multirow{2}{*}{ MS } & \\
\hline & CA1 & CA3 & & \\
\hline Control & $516.3 \pm 19$ & $349.3 \pm 18$ & $154.8 \pm 15$ & $374.6 \pm 37$ \\
\hline MS (Sap) & $335.5 \pm 34$ & $211.8 \pm 28$ & $112 \pm 6.3$ & \\
\hline$M S(I b o)$ & $445.5 \pm 4.9$ & $284.5 \pm 13$ & & $214.0 \pm 12$ \\
\hline
\end{tabular}

The data represent means \pm SEM.

\section{Statistical analysis}

Statistical analysis was performed using SPSS for Windows 11.5.0 (Standard Version, 1982-2002; SPSS Inc., USA). Data of locomotor activity and object exploration in an open field were analyzed by Two-Way Repeated Measures ANOVA with lesion type and session as factors. A one-way ANOVA was performed independently for separate 4 groups on locomotor activity and object exploration data to find significant differences between three sessions. A one-way ANOVA was also used to determine the effect of group on the habituation score data and displacement or object novelty discrimination indexes. Further post hoc comparisons were made using Tukey's tests, where appropriate. Twosample $t$-test was used to compare histological data between control and lesioned groups. All data are presented as mean \pm standard error of the mean. Differences were considered significant when $p<0.05$.

\section{Results}

\section{Histology}

Of the 9 rats that received ibotenic acid lesions targeted at the MS two animals died before the end of the experiment and were excluded from the analysis. In addition, two MS electrolytic lesioned rats were found to have extraseptal damage and were also excluded. In the remaining cohort for which all data are reported, only minimal gliosis was observed. The number of animals in each group was as follows: MS electrolytic $(n=7)$, MS immunotoxic $(\mathrm{n}=9)$, neurotoxic $(\mathrm{n}=7)$ lesioned. Since there were no significant differences $(P>0.05)$ between sham-operated (4 rats) and vehicle-injected rats (8 rats) with regard to activity, habituation and response to a novelty, these groups were combined into a single one, as of now designated as control $(\mathrm{n}=12)$.

Overall, in our experiments electrolytic lesions destroyed on average $69 \pm 4.98 \%$ (mean \pm SEM; range $50-88 \%$ ) of the intact MS. Neurotoxic lesion destroyed most of the medial septal neurons and sham lesion left the medial septum intact: there was significant difference among these groups (Contr: 374.6 \pm 37 ; MS ibo: $214.0 \pm 12 ; t=4.10 ; p=0.015 ; \mathrm{df}=4)$. Examination of the AChE stained sections showed that after injections of 192 IgG-saporin into the MS, animals exhibited significantly less AChE staining in MS ( $t=2.63, P=0.047$, $\mathrm{df}=5$ ) and in hippocampus (CA1 $-t=4.64, P=0.01, \mathrm{df}=4$; CA3 $-t=4.08, P=0.01, \mathrm{df}=4)$ as compared to sections obtained from control animals. Results of our immunohistochemical studies show that after ibotenic acid MSlesions, loss of AChE sensitive neurons in the hippocampus is less than after saporin lesions: differences between these groups in AChE staining in the CAl field of the hippocampus is significant $(t=3.22, P=0.048$, $\mathrm{df}=3$ ), but in the CA3 field of the hippocampus is not significant $(t=2.33, P=0.08, \mathrm{df}=4)$. Interestingly, ibotenic acid lesions appeared to largely spare cholinergic projections to the hippocampus. The results of histological studies are presented in Figures 1 and 2, and Table 1.

\section{Locomotor activity and habituation to the environment}

The Two-Way Repeated Measures ANOVA for the locomotor activity showed no significant effect of group but showed significant effect of session $\left(F_{6,62}=127.656, P<0.0001\right)$ and interaction between group and session $\left(F_{6,62}=7.672, P<0.0001\right)$. No significant difference between sessions was found for the locomotor activity of electrolytic lesion group $\left(F_{2,20}=1.727\right.$, $P=0.206$ ) and a significant difference between sessions for the locomotor activity of control $\left(F_{2,35}=158.52\right.$, $P<0.0001)$, immunotoxic $\left(F_{2,26}=21.272, P<0.0001\right)$ and neurotoxic lesion $\left(F_{2,20}=47.785, P<0.0001\right)$ groups. The results of post hoc analysis of differences between Sessions 1, 2 and 3 are presented in Table 2. 
Table 2. Effect of MS lesions on the locomotor activity and object exploration: post hoc (Tukey HSD) analysis of differences between Sessions 1, 2 and 3 for separate 4 groups.

\begin{tabular}{lccccc}
\hline $\begin{array}{l}\text { Dependent } \\
\text { variable }\end{array}$ & (I) Group & (J) Group & $\begin{array}{c}\text { Mean difference } \\
\text { (I-J) }\end{array}$ & Std. error & Sig \\
\hline \multirow{2}{*}{ HI_LOC } & \multirow{2}{*}{ control } & sap & 7.69 & 8.59 & 0.807 \\
& & ibo & 7.30 & 9.27 & 0.860 \\
& el & $52.15^{*}$ & 9.27 & 0.000 \\
\hline \multirow{2}{*}{ HI_OBJ } & \multirow{2}{*}{ control } & sap & 14.67 & 6.57 & 0.137 \\
& & ibo & $22.19^{*}$ & 7.09 & 0.019 \\
& & el & $23.48^{*}$ & 7.09 & 0.012 \\
\hline
\end{tabular}

* The mean difference is significant at the 0.05 level.

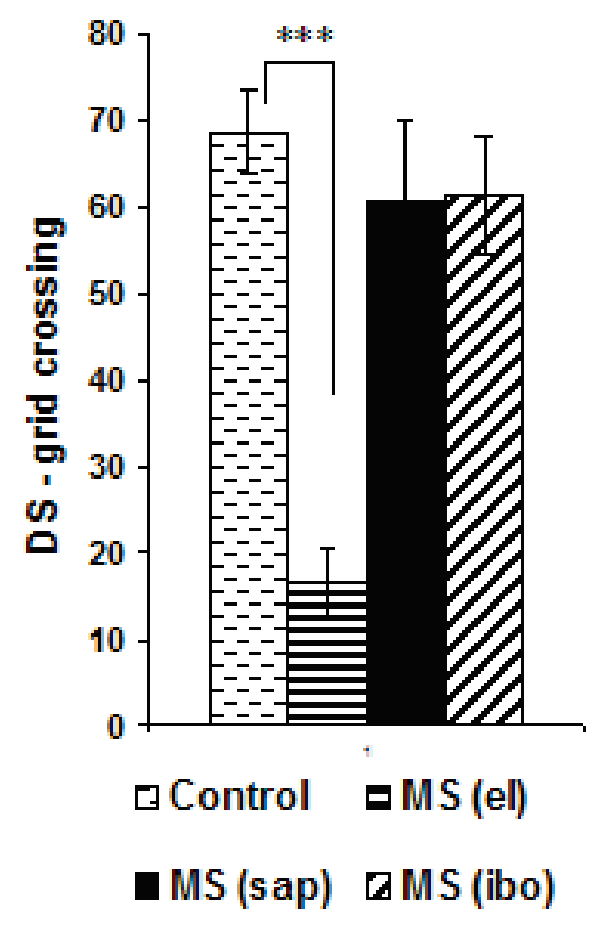

Fig. 3. Effect of MS lesions on the habituation to the environment. Habituation score (difference score - DS) was defined by subtracting the number of crossings in Session 3 from the number of crossings in Session 1. Data are given as mean \pm SEM. $* * * P \leq 0.0001$

The decrease in the number of crossings between Session 1 and Session 3 was taken to be a measure of habituation to the environment. Figure 3 shows effect of MS lesions on the habituation to the environment. Oneway ANOVA for the habituation score data showed significant effect of group $\left(F_{3,34}=11.596, P<0.0001\right)$. Post hoc analysis showed no significant difference between the control and immuno- or neurotoxic lesioned rats $(P>0.5)$ and a significant difference between control and electrolytic lesioned rats $(P<0.0001)$. The results of post hoc analysis of differences between different groups are presented in Table 3.

\section{Object exploration and habituation to the object}

The Two-Way Repeated Measures ANOVA for the object exploration showed significant effect of session $\left(F_{2,62}=19.031, \quad P<0.0001\right)$ and group $\left(F_{3,31}=14.731\right.$, $P<0.0001)$ and significant interaction between group and session $\left(F_{6,62}=3.840, P<0.003\right)$. Post hoc analysis showed a significant difference between the control and electrolytic $(P<0.0001)$ or ibotenic acid lesioned rats $(P<0.004)$ but no significant difference between the control and saporin treated rats and between the electrolytic and ibotenic acid lesioned rats $(P>0.5)$. The ANOVA showed significant differences between sessions for the object exploration of control group $\left(F_{2,35}=29.493\right.$, $P<0.0001)$ and immunotoxic lesion group $\left(F_{2,26}=10.176\right.$, $P<0.001)$. No significant differences was found for the object exploration of electrolytic lesion group $\left(F_{2,20}=0.234\right.$, $P=0.794)$ and neurotoxic lesion group $\left(F_{2,20}=0.297\right.$, $P=0.747)$. The results of post hoc analysis of differences between Sessions 1, 2 and 3 are presented in Table 2.

The decrease in the time spent for exploring four objects between Session 1 and Session 3 was taken to be a measure of habituation to the objects. Figure 4 shows effect of MS lesions on the habituation to the objects. One-way ANOVA for the habituation score data showed significant effect of group $\left(F_{3,34}=5.120, P<0.005\right)$. Post hoc analysis showed no significant difference between the control and immunotoxic lesioned rats $(P>0.5)$ and a significant difference between control and electrolytic or neurotoxic lesioned rats $(P<0.05)$. The results of post hoc analysis of differences between different groups are presented in Table 3. 
Table 3. Effect of MS lesions on the habituation indexes of locomotor activity (HI_LOC) and object exploration (HI_OBJ): post hoc (Tukey HSD) analysis of differences between groups.

\begin{tabular}{|c|c|c|c|c|c|c|c|c|}
\hline \multirow[b]{2}{*}{ Group } & \multicolumn{2}{|c|}{ Sessions } & \multicolumn{3}{|c|}{ Locomotor activity } & \multicolumn{3}{|c|}{ Object exploration } \\
\hline & (I) Ses & (J) Ses & $\begin{array}{c}\text { Mean } \\
\text { difference } \\
(I-J)\end{array}$ & $\begin{array}{l}\text { Std. } \\
\text { error }\end{array}$ & Sig. & $\begin{array}{c}\text { Mean } \\
\text { difference } \\
\text { (I-J) }\end{array}$ & $\begin{array}{l}\text { Std. } \\
\text { error }\end{array}$ & Sig. \\
\hline \multirow[t]{3}{*}{ Control } & 1 & 2 & $54.42 *$ & 4.07 & 0.000 & $20.00 *$ & 3.79 & 0.000 \\
\hline & & 3 & $68.58^{*}$ & & 0.000 & $28.33^{*}$ & & 0.000 \\
\hline & 2 & 3 & $14.17^{*}$ & & 0.004 & 8.33 & & 0.086 \\
\hline \multirow[t]{3}{*}{ MS (sap) } & 1 & 2 & $32.56^{*}$ & 9.34 & 0.005 & $11.33^{*}$ & 3.41 & 0.008 \\
\hline & & 3 & $60.89^{*}$ & & 0.000 & $14.67^{*}$ & & 0.001 \\
\hline & 2 & 3 & $28.33^{*}$ & & 0.015 & 3.33 & & 0.597 \\
\hline \multirow[t]{3}{*}{$M S(i b o)$} & 1 & 2 & $35.14^{*}$ & 6.29 & 0.000 & 3.43 & 7.99 & 0.904 \\
\hline & & 3 & $61.29^{*}$ & & 0.000 & 6.14 & & 0.727 \\
\hline & 2 & 3 & $26.14^{*}$ & & 0.002 & 2.71 & & 0.939 \\
\hline \multirow[t]{3}{*}{$M S(e l)$} & 1 & 2 & 9.43 & 8.87 & 0.548 & 0.71 & 7.66 & 0.995 \\
\hline & & 3 & 16.43 & & 0.182 & 4.86 & & 0.804 \\
\hline & 2 & 3 & 7.00 & & 0.714 & 4.14 & & 0.852 \\
\hline
\end{tabular}

* The mean difference is significant at the 0.05 level.

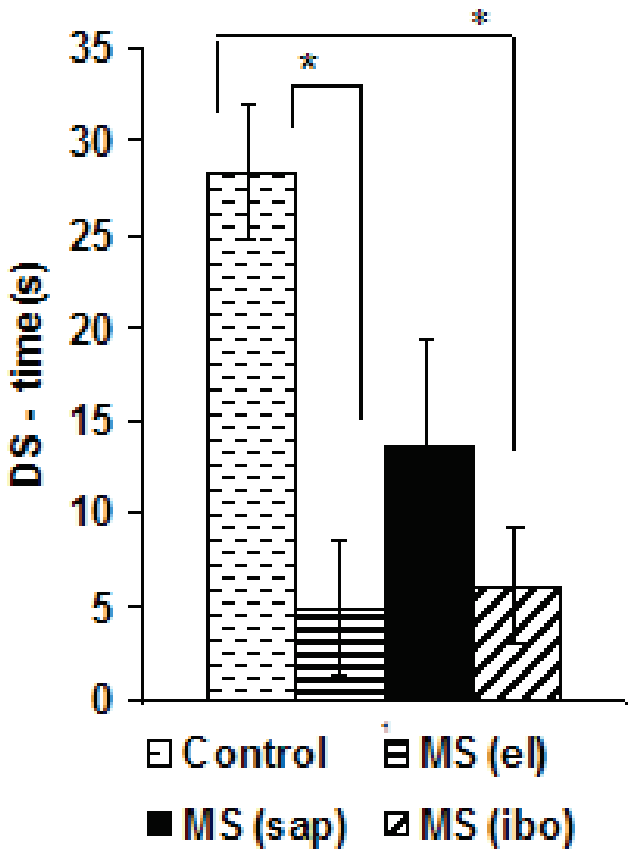

Fig. 4. Effect of MS lesions on the habituation to the objects. The habituation score (difference score - DS) was defined by subtracting the time spent for exploring objects in Session 3 from the time spent for the same objects during Session 1. Data are given as mean \pm SEM. * $P \leq 0.05$

\section{Detection of spatial novelty}

Figure 5A shows the difference between the responses to spatial change (defined by DI) at Session 4 by control and MS lesioned rats. One-way ANOVA for the displacement discrimination index revealed significant effect of group $\left(F_{3,34}=11.821, P<0.0001\right)$. Post hoc analysis showed a significant difference between the control and electrolytic lesioned rats $(P<0.0001)$ and no significant difference between the control and MS immuno- or neurotoxic lesioned rats $(P>0.2)$. The mean discrimination indexes in control $\left(\mathrm{DI}_{\mathrm{D}}=0.69 \pm 0.023\right)$ immuno- $\quad\left(\mathrm{DI}_{\mathrm{D}}=0.65 \pm 0.034\right) \quad$ or neurotoxic $\left(\mathrm{DI}_{\mathrm{D}}=0.6 \pm 0.027\right)$ lesioned rats were significantly higher from chance performance $(t=8.11, P<0.0001, t=4.5$, $P<0.002, \quad t=3.87, \quad P<0.008$ respectively). The mean discrimination index $\left(\mathrm{DI}_{\mathrm{D}}\right)$ in electrolytic lesioned rats $\left(\mathrm{DI}_{\mathrm{D}}=0.43 \pm 0.051\right)$ did not differ significantly from chance level $(t=1.229, P>0.2)$.

\section{Detection of object change}

Figure 5B shows the responses to the object novelty (defined by DI) at Session 5 by control and MS lesioned rats. One-way ANOVA for the object novelty discrimination index revealed no significant effect of group $\left(F_{3,34}=1.632, P>0.2\right)$. The mean discrimination indexes in control $\left(\mathrm{DI}_{\mathrm{N}}=0.65 \pm 0.021\right)$, immunotoxic $\left(\mathrm{DI}_{\mathrm{N}}=0.62 \pm 0.039\right)$, neurotoxic $\left(\mathrm{DI}_{\mathrm{N}}=0.64 \pm 0.036\right)$ and electrolytic $\left(\mathrm{DI}_{\mathrm{N}}=0.58 \pm 0.034\right)$ lesioned rats were significantly higher from chance performance $(t=7.0$, $P<0.0001, t=3.212, P<0.012, t=3.873, P<0.008, t=2.521$, $P<0.045$ respectively). 


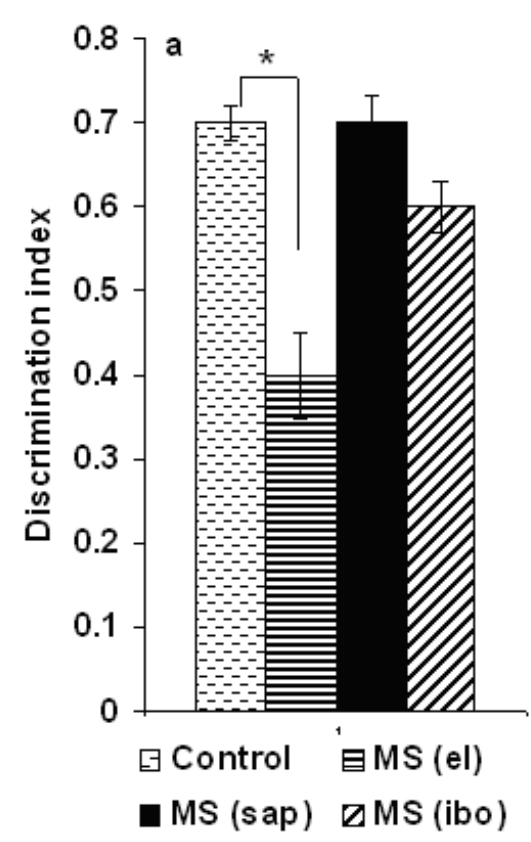

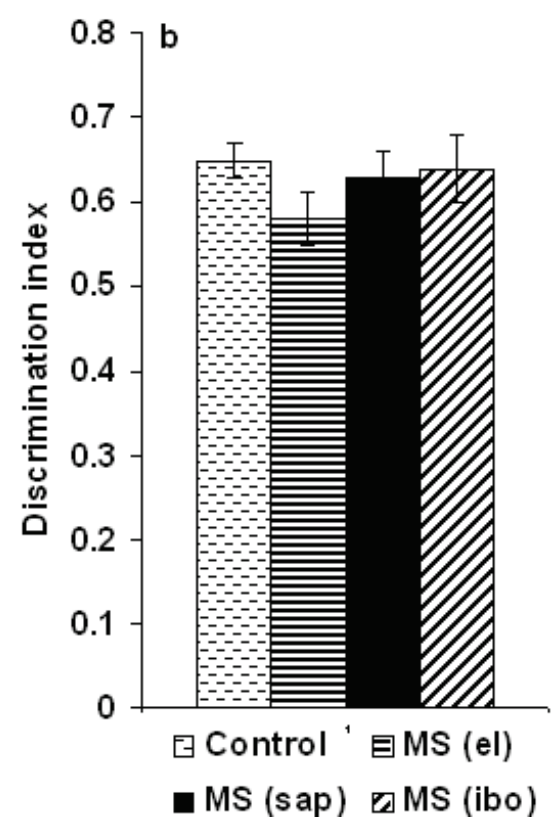

Fig. 5. Effect of MS lesions on the behavioral response to the spatial change (a) and object change (b). The histograms represent difference between spatial change scores or object change scores defined by discrimination indexes (DIs). Displacement discrimination index (DI $)$ was calculated as: $\mathrm{DI}_{D}=\mathrm{t}_{\mathrm{D}} /\left(\mathrm{tN}_{\mathrm{D}}\right.$ $\left.+t_{D}\right)$, where $t_{D}=$ exploration time of the displaced objects and $\mathrm{tN}_{\mathrm{D}}=$ mean exploration time of the non-displaced objects. The object novelty discrimination index, $\mathrm{DI}_{\mathrm{N}}$ was calculated as: $D I_{N}=t_{N} /\left(t_{F}+t_{N}\right)$, where $t_{N}=$ exploration time of the novel object and $t_{F}=$ mean exploration time of the familiar objects. Data are given as mean \pm SEM. * $P \leq 0.05$

\section{Discussion}

This experiment compares three types of MS lesions: electrolytic lesions that destroy cells and fibers of passage, neurotoxic - ibotenic acid lesions that spare fibers of passage but predominantly affect the septal noncholinergic neurons, and immunotoxin - 192 IgGsaporin infusions that only eliminate cholinergic neurons. The results provide new insights into what aspects of MS circuitry are involved in exploratory behavior, habituation and recognition memory.

The results of present study indicate that control rats as well as all MS lesioned rats clearly react to the object novelty by exploring the new object more than familiar ones. These findings point to that the object novelty detection may be due to a mechanism that is not dependent on the MS. These findings are in line with several investigations showing that hippocampal or fornix lesions produce no effect in object recognition (Ennaceur et al. 1996, 1997, Langston and Wood 2010). Similarly, Kornecook et al. (1999) found that rats with electrolytic lesions of the MS and diagonal band nuclei of the basal forebrain were not impaired on a simple two-choice object-discrimination task. In this study lesions were considerably larger and consistently included not only the MS, but also sizable portions of both the vertical and horizontal divisions of the diagonal band nuclei. In another research Kelsey and Vargas (1993) found that rats with small lesions of the MS were impaired at performing a spatial delayed nonmatching-to-sample task taxing the spatial recognition memory, but displayed no deficits on a similar nonspatial task. In contrast to the current study, Myhrer (1989) found that MS electrolytic lesioned rats reduced preference for novelty. There are many possible causes for these differing results. First, in Myhrer's study the lesioned rats displayed decreased exploration of both neutral and novel objects. It may be suggested that the rats failed to show preference for novelty partly because of an initially lower level of exploration relative to normal animals. Second, there are differences in the behavioral paradigms used: in contrast to our study the rats were allowed to explore the empty apparatus. It is interesting to note, that in some studies presentation of the objects is preceded with habituation session/s in the empty open field (Paban et al. 2005). Because no rewarding or aversive stimulation is used during recognition training, the learning occurs under conditions of relatively low stress or arousal (Ennaceur and Delacour 1988). Thus, rats habituated to the training apparatus would be expected to be less aroused by recognition training than rats not given prior habituation training. Also, should be taken into account differences in the open field size, number of objects used and intersession intervals. In general, procedural differences make it difficult to compare the results of different laboratories.

Dysfunction of the hippocampus induced by lesions of the MS therefore may result in behavioral effects similar to those of hippocampal lesions. Lesions of the fimbria-fornix interfere both with learning and memory tasks and with generation of the hippocampal theta rhythm in rats (Brito and Brito 1990). However, the 
role of the hippocampus in recognition tasks has been controversial: some investigations showed impaired recognition performance (Broadbent et al. 2004, Clark et al. 2000, Lee et al. 2005, Paban et al. 2005) and some did not (Gaskin et al. 2003, Winters et al. 2004). In the Broadbent's study the object recognition memory was impaired only after nearly complete hippocampal lesions (75-100\%) and was entirely spared by smaller lesions, while the spatial memory is severely impaired (Broadbent et al. 2004). Consequently it is not unexpected, that the selective damage to a subset of hippocampal afferents does not produce an effect equivalent to the effect of complete hippocampal lesion. Also, there may be conditions under which hippocampal lesions will produce reliable impairments. For example: rats with hippocampal lesions failed to display a preference when the sampleexposure context and test context were different. The performance of control rats was unaffected by the change in context (O'Brien et al. 2006). The results of the present study are in accordance with these findings: in our experiments the sample-exposure context and test context were the same.

It is interesting to note, that while the control rats showed the increase in exploration when a familiar object was moved to an unfamiliar location, the rats with electrolytic lesions of MS did not demonstrate such enhanced exploration. The present results reveal that electrolytic lesions of the MS disrupt spatial recognition memory and that the MS is more specialized for detecting spatial changes than changes in objects in the environment. In general, our data disprove the possibility that the deficit in detecting the displaced objects in the electrolytic MS lesioned rats might be due to a decrease in the natural tendency to explore novelty: the rats were normal in exploring the novel object introduced to the environment. Because MS electrolytic lesion had no effect in the detection of object novelty, the deficit observed in the case of spatial novelty could not be a consequence of disrupted attentional processing: such disruption would compromise the performance of both tasks. According to the data obtained it is possible to suggest that the MS is necessary for normal spatial recognition memory and the processing of information about the spatial environment and that the object and spatial recognition memory could be mediated by different neuronal substrates. Much evidence has indicated a critical role for the perirhinal cortex in familiarity discrimination of objects (Ennaceur et al. 1996, Brown and Aggleton 2001) while the studies examining the effects of hippocampal or nonselective MS lesions have reported the impairment in spatial recognition memory (Poucet 1989, Kelsey and Vargas 1993, Broadbent et al. 2004).

However, electrolytic lesions are non-specific. Damage as the result of the MS electrolytic lesions technique compromises cell bodies, afferents fibers, efferent fibers, and fibers of passage. Therefore the behavior impairments after electrolytic lesions of MS may depend on compromised function in any of these systems. The septohippocampal projections are predominantly cholinergic and GABAergic (Rye et al. 1984) and electrolytic MS lesions necessarily result in loss of both cholinergic and noncholinergic septohippocampal projections. The current study using cell specific lesion techniques provides insight into the role of the cholinergic and noncholinergic septohippocampal projections in organizing exploratory behavior and recognition memory.

Results of our immunohistochemical studies show that after ibotenic acid MS-lesions, loss of AChE sensitive neurons in the hippocampus is less than after saporin lesions. These results suggest that Ibotenic acid lesions of the MS spare most of the cholinergic MS neurons and the cholinergic input into the hippocampus is predominantly intact. Similar neurochemical effects of ibotenic acid in the MS have been reported by Cahill and Baxter (2001) and in the nucleus basalis by Burk and Sarter (2001). Such selective effect of neurotoxins may be due to the use of low doses and/or the neuronal network organization of the target structure. The ibotenic acid may also damage other noncholinergic neurons in the MS, and we refer to the damage produced by intraseptal ibotenic acid as noncholinergic because of this uncertainty.

In our study MS ibotenic acid lesioned rats showed an increase in their exploratory behavior to the objects. This phenomenon did not depend on changes of locomotor activity since no effect was observed in this group of rats compared with control ones. In addition, ibotenic acid lesioned rats were impaired in habituating to the objects in the repeated spatial environment throughout the experiment, but they were normal in detecting the spatial or object novelty: loss of noncholinergic septohippocampal projections induced by ibotenic acid injection to the MS disrupts the organization of exploratory behavior but does not disrupt the function of the hippocampus to a sufficient extent to impair recognition memory. One important aspect that has to be 
considered is that the rats with electrolytic or ibotenic acid lesions failed to habituate to the unchanged objects but they were normal in detecting the object novelty. From the behavior of the control animals, it appears that the various objects evoked different preferences for novelty. It may be suggested that the particular novel object used was more attractive to the rats by itself, but in our preliminary study have been chosen the objects which can be easily discriminated by the naïve animals but should not be differentially preferred. In our experiments the rats with electrolytic or ibotenic acid lesions showed the decreased habituation to the objects versus control rats. However the comparison of the results of the object exploration in sessions 1 and 3 revealed the reduced exploration in the session 3 (however this reduction is the less then in control animals). It may be suggested that despite decreased habituation, the ability to encode or store a representation of the context in which the objects are encountered are to a certain extent spared, which is sufficient for detection of changes in the environment.

The introduction of the immunotoxin 192 IgGsaporin appears to allow for more selective cholinergic lesions than electrolytic lesion methods or the injection of neurotoxins (Walsh and Opello 1992). At present, the 192 IgG-SAP immunotoxin is considered an effective tool for selectively eliminating cholinergic neurons from the basal forebrain, and it has proven to be very useful for investigating the role of the cholinergic system in cognition (for a review, see Wrenn and Wiley 1998, McGaughy et al. 2000). The results showed that after injections of $192 \mathrm{IgG}$-saporin into the MS, animals exhibited significantly less AChE staining in hippocampus as compared to sections obtained from control animals. Our immunohistochemical data are in line with a growing body of findings showing that intraseptal 192 IgG-saporin injections induce extensive loss of AChE sensitive neurons in the hippocampus.

In present study 192 IgG-saporin lesioned rats were intact in habituating to the repeated spatial environment throughout the experiment and they were normal in detecting the changes in locations of familiar objects. These findings indicate that septohippocampal cholinergic projections are not necessary for detection of spatial changes and that the deficits observed after septal electrolytic lesions cannot be accounted solely to the loss of hippocampal ACh. Studies examining the effects of selective lesion techniques have resulted in both impairments (Pang et al. 2001, Lamprea et al. 2003, Lehmann et al. 2003, Paban et al. 2005, Cai et al. 2012) and intact performance on a variety of spatial tasks (Baxter and Gallagher 1996, Winters and Dunnett 2004). However, the interpretation of the deficits after immunolesions as consequent to selective loss of cholinergic MS neurons is sometimes questionable. In many cases, these studies have used high doses of 192 IgG-saporin and/or injection protocols that may compromise noncholinergic MS neurons. Paban et al. (2005) showed that after cholinergic lesions the most robust behavioral deficit only appeared at the longest post-operative time (days 425-443). At the long postoperative testing times behavioral deficits would be linked to the decompensation of non-cholinergic neuronal systems. Furthermore, when impairments are seen in memory tasks after cholinergic lesions, they may be caused by an influence on a nonmnemonic process. The study by Paban et al. (2005) seems of particular interest here. They demonstrated that 192 IgG-SAP-treated rats tested at the earliest post-lesion time (days 26-47) exhibited a deficit but only if the retention interval was as brief as $1 \mathrm{~min}$. This pattern of impairment tends to reflect a disruption of either short-term memory or some other non-mnemonic process such as attention. The latter hypothesis is in line with several investigations suggesting that impairments in performance following cholinergic lesions are attentional in nature (Baxter 2001, Lehmann et al. 2003). Memory tasks that include an increased burden on attention should be more sensitive to loss of basal forebrain cholinergic neurons (e.g., Turchi and Sarter 2000). Future work needs to be done to further explore and differentiate the specific effects of selective lesions of cholinergic and noncholinergic septohippocampal projections in tests taxing attention and spatial memory function.

The results of present study indicate that spatial recognition memory is affected only by electrolytic lesions but not saporin or ibotenic lesions. It may be suggested that the effects of electrolytic lesions can be caused by the lesion of the fibers of passage, but it is impossible to exclude, that it is related to the combined lesions of the cholinergic and GABAergic septohippocampal projection neurons. It is not surprising that selective damage to a subset of septohippocampal afferents does not produce an effect equivalent to that of nonselective lesion of MS. It is interesting to note that 192 IgG-saporin or kainic-acid-induced lesions do not produce spatial working memory deficits or make only mild impairment (Pang et al. 2001). In contrast, combined lesions of the cholinergic and GABAergic 
septohippocampal projection neurons produce severe deficits (Pang et al. 2001). To determine more definitively whether septohippocampal projection neurons are required for the recognition memory it would be ideal to produce in future combined lesions of the cholinergic and GABA-ergic septohippocampal projection neurons using 192 IgG-saporin and a new GABAergic immunotoxin GABA-transporter-saporin (GAT1-SAP).

\section{Conclusion}

Our results together with the short review of a literature, indicate that: (i) the deficits after nonselective damage of MS are limited to a subset of cognitive processes dependent on the hippocampus, (ii) MS is substantial for spatial, but not for object recognition memory - the object recognition memory can be supported outside the septohippocampal system; (iii) the selective loss of septohippocampal cholinergic or noncholinergic projections does not disrupt the function of the hippocampus to a sufficient extent to impair spatial recognition memory; (iv) there is dissociation between the two major components (cholinergic and noncholinergic) of the septohippocampal pathway in exploratory behavior assessed in the open field - the memory exhibited by decrements in exploration of repeated object presentations is affected by either electrolytic or ibotenic lesions, but not saporin and this behavior relies on non-cholinergic MS cells.

\section{Conflict of Interest}

There is no conflict of interest.

\section{Acknowledgements}

This research has been fulfilled by financial support of Shota Rustaveli National Science Foundation of the Ministry of Education and Science of Georgia (Grant $\# 13 / 10)$.

\section{References}

BALDERAS I, MORIN JP, RODRIGUEZ-ORTIZ CJ, BERMUDEZ-RATTONI F: Muscarinic receptors activity in the perirhinal cortex and hippocampus has differential involvement in the formation of recognition memory. Neurobiol Learn Mem 97: 418-424, 2012.

BARBOSA FF, DE OLIVEIRA PONTES IM, RIBEIRO S, RIBEIRO AM, SILVA RH: Differential roles of the dorsal hippocampal regions in the acquisition of spatial and temporal aspects of episodic-like memory. Behav Brain Res 232: 269-277, 2012.

BARKER GR, WARBURTON EC: When is the hippocampus involved in recognition memory? J Neurosci 31: 10721$10731,2011$.

BAXTER MG: Effects of selective immunotoxic lesions on learning and memory. Methods Mol Biol 166: 249-265, 2001.

BAXTER MG, GALLAGHER M: Intact spatial learning in both young and aged rats following selective removal of hippocampal cholinergic input. Behav Neurosci 110: 460-467, 1996.

BOLIVAR V, FLAHERTY L: A region on chromosome 15 controls intersession habituation in mice. J Neurosci 23 : 9435-9438, 2003.

BRITO GN, BRITO LS: Septohippocampal system and the prelimbic sector of frontal cortex: a neuropsychological battery analysis in the rat. Behav Brain Res 36: 127-146, 1990.

BROADBENT NJ, SQUIRE LR, CLARK RE: Spatial memory, recognition memory, and the hippocampus. Proc Natl Acad Sci U S A 101: 14515-14520, 2004.

BROWN MW, AGGLETON JP: Recognition memory: What are the roles of the perirhinal cortex and hippocampus? Nat Rev Neurosci 2: 51-61, 2001.

BURK JA, SARTER M: Dissociation between the attentional functions mediated via basal forebrain cholinergic and GABAergic neurons. Neuroscience 105: 899-909, 2001.

CAHILL JF, BAXTER MG: Cholinergic and noncholinergic septal neurons modulate strategy selection in spatial learning. Eur J Neurosci 14: 1856-1864, 2001.

CAI L, GIBBS RB, JOHNSON DA: Recognition of novel objects and their location in rats with selective cholinergic lesion of the medial septum. Neurosci Lett 506: 261-265, 2012. 
CHANG Q, GOLD PE: Switching memory systems during learning: changes in patterns of brain acetylcholine release in the hippocampus and striatum in rats. J Neurosci 23: 3001-3005, 2003.

CHOI DW, KOH JY, PETERS S: Pharmacology of glutamate neurotoxicity in cortical cell culture: attenuation by NMDA antagonists. $J$ Neurosci 8: 185-196, 1988.

CLARK RE, ZOLA SM, SQUIRE LR: Impaired recognition memory in rats after damage to the hippocampus. J Neurosci 20: 8853-8860, 2000.

DASHNIANI MG, BESELIIA GV, MAGLAKELIDZE GA, BURDZHANADZE MA, CHKHIKVISHVILI NTs: Effects of the selective lesions of cholinergic septohippocampal neurons on different forms of memory and learning process. Georgian Med News 166: 81-85, 2009.

DIX SL, AGGLETON JP: Extending the spontaneous preference test of recognition: evidence of object-location and object-context recognition. Behav Brain Res 99: 191-200, 1999.

DUNNETT SB, EVERITT BJ, ROBBINS TW: The basal forebrain-cortical cholinergic system: interpreting the functional consequences of excitotoxic lesions. Trends Neurosci 14: 494-501, 1991.

ENNACEUR A, DELACOUR J: A new one-trial test for neurobiological studies of memory in rats. 1: Behavioral data. Behav Brain Res 31: 47-59, 1988.

ENNACEUR A, NEAVE N, AGGLETON JP: Neurotoxic lesions of the perirhinal cortex do not mimic the behavioural effects of fornix transection in the rat. Behav Brain Res 80: 9-25, 1996.

ENNACEUR A, NEAVE N, AGGLETON JP: Spontaneous object recognition and object location memory in rats, the effects of lesions in the cingulate cortices, the medial prefrontal cortex, the cingulum bundle and the fornix. Exp Brain Res 113: 509-519, 1997.

FRICK KM, KIM JJ, BAXTER MG: Effects of complete immunotoxin lesions of the cholinergic basal forebrain on fear conditioning and spatial learning. Hippocampus 14: 244-254, 2004.

GASKIN S, TREMBLAY A, MUMBY DG: Retrograde and anterograde object recognition in rats with hippocampal lesions. Hippocampus 13: 962-969, 2003.

JARRARD LE: On the use of ibotenic acid to lesion selectively different components of the hippocampal formation. J Neurosci Methods 29: 251-259, 1989.

KELSEY JE, VARGAS H: Medial septal lesions disrupt spatial, but not nonspatial, working memory in rats. Behav Neurosci 107: 565-574, 1993.

KIRBY BP, RAWLINS JN: The role of the septo-hippocampal cholinergic projection in T-maze rewarded alternation. Behav Brain Res 143: 41-48, 2003.

KORNECOOK TJ, KIPPIN TE, PINEL JP: Basal forebrain damage and object-recognition in rats. Behav Brain Res 98 : 67-76, 1999.

LAMPREA MR, CARDENAS FP, SILVEIRA R, WALSH TJ, MORATO S: Effects of septal cholinergic lesion on rat exploratory behavior in an open-field. Braz J Med Biol Res 36: 233-238, 2003.

LANGSTON RF, WOOD ER: Associative recognition and the hippocampus: differential effects of hippocampal lesions on object-place, object-context and object-place-context memory. Hippocampus 20: 1139-1153, 2010.

LECOURTIER L, DE VASCONCELOS AP, LEROUX E, COSQUER B, GEIGER K, LITHFOUS S, CASSEL JC: Septohippocampal pathways contribute to system consolidation of a spatial memory: sequential implication of GABAergic and cholinergic neurons. Hippocampus 21: 1277-1289, 2011.

LEE I, HUNSAKER MR, KESNER RP: The role of hippocampal subregions in detecting spatial novelty. Behav Neurosci 119: 145-153, 2005.

LEHMANN O, GROTTICK AJ, CASSEL JC, HIGGINS GA: A double dissociation between serial reaction time and radial maze performance in rats subjected to $192 \mathrm{IgG}$-saporin lesions of the nucleus basalis and/or the septal region. Eur J Neurosci 18: 651-666, 2003.

MCGAUGHY J, EVERITT BJ, ROBBINS TW, SARTER M: The role of cortical cholinergic afferent projections in cognition: impact of new selective immunotoxins. Behav Brain Res 115: 251-263, 2000.

MESULAM MM, MUFSON EJ, WAINER BH, LEVEY AI: Central cholinergic pathways in the rat: an overview based on an alternative nomenclature (Ch1-Ch6). Neuroscience 10: 1185-1201, 1983.

MYHRER T: Exploratory behavior and reaction to novelty in rats: effects of medial and lateral septal lesions. Behav Neurosci 103: 1226-1233, 1989. 
NIEWIADOMSKA G, BAKSALERSKA-PAZERA M, GASIOROWSKA A, MIETELSKA A: Nerve growth factor differentially affects spatial and recognition memory in aged rats. Neurochem Res 31: 1481-1490, 2006.

O'BRIEN N, LEHMANN H, LECLUSE V, MUMBY DG: Enhanced context-dependency of object recognition in rats with hippocampal lesions. Behav Brain Res 170: 156-162, 2006.

OKADA K, OKAICHI H: Functional cooperation between the hippocampal subregions and the medial septum in unreinforced and reinforced spatial memory tasks. Behav Brain Res 209: 295-304, 2010.

PABAN V, JAFFARD M, CHAMBON C, MALAFOSSE M, ALESCIO-LAUTIER B: Time course of behavioral changes following basal forebrain cholinergic damage in rats: environmental enrichment as a therapeutic intervention. Neuroscience 132: 13-32, 2005.

PANG KC, NOCERA R, SECOR AJ, YODER RM: GABAergic septohippocampal neurons are not necessary for spatial memory. Hippocampus 11: 814-827, 2001.

PAXINOS G, WATSON C: The Rat Brain in Stereotaxic Coordinates, 4th ed. Academic Press, San Diego, CA, 1998.

PETERSON GM, SHURLOW CL: Morphological evidence for a substance P projection from medial septum to hippocampus. Peptides 13: 509-517, 1992.

POUCET B: Object exploration, habituation, and response to a spatial change in rats following septal or medial frontal cortical damage. Behav Neurosci 103: 1009-1016, 1989.

RYE DB, WAINER BH, MESULAM MM, MUFSON EJ, SAPER CB: Cortical projections arising from the basal forebrain: a study of cholinergic and noncholinergic components employing combined retrograde tracing and immunohistochemical localization of choline acetyltransferase. Neuroscience 13: 627-643, 1984.

SOTTY F, DANIK M, MANSEAU F, LAPLANTE F, QUIRION R, WILLIAMS S: Distinct electrophysiological properties of glutamatergic, cholinergic and GABAergic septohippocampal neurons: novel implications for hippocampal rhythmicity. J Physiol 551: 927-943, 2003.

TURCHI J, SARTER M: Cortical cholinergic inputs mediate processing capacity: effects of 192 IgG-saporin-induced lesions on olfactory span performance. Eur J Neurosci 12: 4505-4514, 2000.

WALSH TJ, OPELLO KD: Neuroplasticity, the aging brain, and Alzheimer's disease. Neurotoxicology 13: 101-110, 1992.

WINTERS BD, DUNNETT SB: Selective lesioning of the cholinergic septo-hippocampal pathway does not disrupt spatial short-term memory: a comparison with the effects of fimbria-fornix lesions. Behav Neurosci 118: 546$562,2004$.

WINTERS BD, FORWOOD SE, COWELL RA, SAKSIDA LM, BUSSEY TJ: Double dissociation between the effects of peri-postrhinal cortex and hippocampal lesions on tests of object recognition and spatial memory: heterogeneity of function within the temporal lobe. J Neurosci 24: 5901-5908, 2004.

WRENN CC, WILEY RG: The behavioral functions of the cholinergic basal forebrain: lessons from 192 IgG-saporin. Int J Dev Neurosci 16: 595-602, 1998.

YODER RM, PANG KC: Involvement of GABAergic and cholinergic medial septal neurons in hippocampal theta rhythm. Hippocampus 15: 381-392, 2005. 